\title{
Las posibilidades de internet en la reconfiguración de la red social primaria del adulto mayor, fracturada por efecto de la migración*
}

\author{
El caso de los adultos mayores con parientes migrantes en 6 municipios \\ del departamento del Quindío
}

\author{
Recibido: 2015-02-20 -Enviado a pares: 2015-03-10 \\ Aprobado por pares: 2015-04-05 Aceptado: 2015-04-30 \\ Lucero Giraldo Marín ** \\ Sandra Leal Larrarte**x \\ Lilia Inés López Cardozo****
}

\section{Resumen}

El presente artículo muestra cómo Internet se convierte en una herramienta de comunicación importante para reconfigurar la red social primaria del adulto mayor, fracturada por efecto de la migración.

En la investigación que le da origien, se pudo constatar que hoy esta red se encuentra dividida en red primaria natural, conformada por los parientes y amigos con los que comparte el día a día, y red primaria virtual, integrada por los parientes migrantes.

El escrito centra su interés en presentar las características de la red primaria virtual en la que el adulto mayor se vio impelido a participar para satisfacer sus necesidades comunicacionales. Esta red cumple la función de mantener, pero no de extender, su red social primaria.

Los resultados dan cuenta de 4 aspectos: las competencias digitales desarrolladas por el adulto mayor, la estructura, las funciones y los atributos de vínculo de la red primaria virtual. Para ello se acudió a autores como Cabrera, Castell; Madarriaga, Abello \& Sierra; Narváez A.; Soto, Navarro \& Sánchez; Tovar E Villarraga. La investigación fue de tipo etnográfico, con enfoque cualitativo. Se aplicaron entrevistas semiestructuradas. Para el tratamiento de la información se utilizó la teoría Fundamentad, de Strauss \& Corbin (2002).

Palabras clave: adulto mayor, Internet, migración, red personal, redes sociales.

\footnotetext{
Este artículo surge de los resultados de la investigación "Internet y red personal del adulto mayor de los municipios de Filandia, Armenia, Calarcá, Quimbaya, Salento y Montenegro - Quindío, con parientes migrantes", realizada durante el año 2012, por docentes del Programa de Comunicación Social y Periodismo de la Universidad del Quindío, en la línea de Comunicación y Medios, financiada por la misma institución.

** Docente Universidad del Quindío, Programa de Comunicación Social-Periodismo. Magíster en Comunicación Educativa de la Universidad Tecnológica de Pereira. Coordinadora de Investigación del programa de Comunicación Social-Periodismo, docente de la asignatura de Comunicación para el Desarrollo, asesora de trabajos de grado y de intervención social en la Universidad del Quindío. Ponente en eventos nacionales e internacionales (Colombia). lgiraldo@uniquindio.edu.co

*** Magíster en Ciencias de la Comunicación con énfasis en sociosemiótica, título otorgado por la Universidad del Zulia (Venezuela). Ha sido ponente nacional e internacional en varias ocasiones, jurado y tutora de trabajos de grado para pregrado. salale34@yahoo.com.ar

**** Docente Universidad del Quindío, Programa Comunicación social-Periodismo. Gerontóloga Universidad del Quindío, magíster en Educación Universidad, Pontificia Javeriana-Universidad de Caldas. Se ha desempeñado como directora de los programas académicos de Gerontología y Comunicación Social-Periodismo en la Universidad del Quindío y como docente del área investigativa en las asignaturas: Proyecto de Grado I-II, Seminario de Investigación Social, Seminario Taller de Investigación, Metodología de la Investigación, entre otros. liliai@uniquindio.edu.co
} 


\title{
Internet's possibilities in the reconfiguration of the primary social network of the elderly, which is fractured due to the effect of migration
}

The case of the elderly with migrant relatives in 6 municipalities of Quindío.

\begin{abstract}
This article shows how Internet becomes an important communication tool for reconfiguring the primary social network of the elderly, which is fractured due to the effect of migration.

In the research that generates this article it was possible to confirm that this network is currently divided into the primary natural network, formed by the relatives and friends with whom they share their daily life, and the primary virtual network, which is formed by the migrant relatives.

The paper focuses its interest on presenting the characteristics of the primary virtual network, in which the elderly found themselves impelled to participate in order to satisfy their communicational needs. This network fulfills the function of maintaining, although not expanding, their primary social network.

The results show four aspects: the digital competences developed by the elderly, the structure, the functions, and the bonding attributes of the primary virtual network. For conducting this study, the work of authors such was Cabrera; Castell; Madarriaga, Abello \& Sierra; Narváez A.; Soto, Navarro \& Sánchez; Tovar \& Villarraga was consulted.

It was an ethnographic research with a qualitative approach. Semi-structured interviews were performed. The fundamental theory of Strauss \& Corbin (2002) was used for processing the information.

Key words: elderly, Internet, migration, personal network, social networks.
\end{abstract}

\section{As posibilidades da internet na reconfiguração da rede social primária dos idosos, fraturada pelo efeito da migração O caso dos idosos com parentes migrantes em seis municipios do Quindío}

\section{Resumo}

Este artigo mostra como a internet se torna um instrumento de comunicação importante para reconfigurar a rede social primária dos idosos, fraturada pelo eifeito da migração.

Na pesquisa que lhe dá origem, se pode constatar que atualmente esta rede se encontra dividida em: rede primária natural, formada por parentes e amigos com os quais convivem e rede primária virtual, integrada por parentes migrantes.

O artigo apresenta as características da rede primária virtual onde o idoso se sentiu obrigado a participar para satisfazer suas necessidades de comunicação. Esta rede tem a função de manter, mas não de ampliar, a sua rede social primária.

Os resultados mostram quatro aspectos: as competências digitais desenvolvidad pelos idosos, a estrutura, as funções e os atributos do vínculo da rede virtual primária. Para esta análise, se contaram com autores como: Cabrera, Castell; Madarriaga, Abello \& Sierra; Narváez A.; Soto, Navarro \& Sánchez; Tovar \& Villarraga.

A pesquisa aplicada foi etnográfica qualitativa. Foram feitas entrevistas semi-estruturadas e a Teoria Fundamentada de Strauss \& Corbin (2002) foi utilizada para analisar a informação.

Palabras chave:Idoso, Internet, migração, rede pessoal, redes sociais. 


\section{Introducción}

La temática que abordó la investigación responde a inquietudes sobre tres procesos sociales que afectan la realidad regional, nacional y mundial: migración, redes sociales y calidad de vida de los adultos mayores, los cuales confluyen de tal manera, que terminan por marcar algunas de las particularidades del mundo contemporáneo.

El fenómeno migratorio siempre presente en las sociedades humanas, y se reviste en la época actual de múltiples complejidades tanto por su magnitud como por las lógicas que lo promueven o lo tratan de controlar. Interesó en esta investigación en cuanto a su relación y consecuencias sobre la calidad de vida del adulto mayor, puesto que como proceso social causa la ruptura de la red primaria, conformada por familiares y amigos cercanos, que son su soporte vital, situación que lo enfrenta a nuevos retos personales, sociales y tecnológicos, en la búsqueda de recomponer la red fracturada.

La red social primaria, ante una ruptura, como toda red de los sistemas vivos, busca rehacerse, equilibrarse, sanarse, y en ese proceso cambia el entramado original, por ejemplo, cuando se sufre alguna herida en la piel, queda una cicatriz; así, cuando un integrante de la red primaria migra, esta se obliga a recomponerse añadiendo nuevos contactos o encontrando nuevas maneras de comunicación para recrear el vínculo perdido.

En la conjunción de esas dos rupturas: la del migrante y la del adulto mayor que ve migrar, aparece como elemento mediador la Internet que, como medio de comunicación interactivo y al combinar formas de participación sincrónicas y asincrónicas, ha revolucionado los modos de la comunicación del sistema humano. Esta permite una amplia variedad de posibilidades comunicativas, sin presencia física, entre ellas las redes sociales electrónicas que funcionan como potenciadores, además de extensores, de los vínculos humanos, y facilitan el contacto entre individuos.

Por lo anterior, se plantea que Internet ha aumentado la capacidad de resiliencia del conjunto social, término que adoptado de las ciencias naturales, se refiere a la capacidad de un material para retomar su forma luego de haber sido sometido a altas presiones que lo hubieren deformado. Soto, Montero \& Sánchez (2006) aclaran que "resiliencia humana [... es la capacidad de un individuo o de un sistema social de vivir bien y desarrollarse positivamente, a pesar de las difíciles condiciones de vida y más aún, de salir fortalecidos y ser transformados por ellas" (p. 5).

De esa manera, a través de Internet se enfrenta la disgregación de las comunidades humanas por problemas políticos y/o económicos y se encuentra una vía para superar el dolor de la separación, de la necesidad del otro. Internet llegó en el momento preciso dentro de la historia humana, pues no solo le permite al individuo socializar, sino que implica una unión espacio-tiempo que reunifica las prácticas sociales y familiares abandonadas a favor de la seguridad económica, física y/o de las libertades y sueños individuales.

De la inquietud por los procesos mencionados surgieron las preguntas que orientaron la investigación que sirvió de base al presente artículo: ¿Cómo contribuye Internet en la 
reconfiguración de la red social primaria del adulto mayor, fracturada por efecto de la migración? ¿Cómo enfrentan los adultos mayores el reto tecnológico de comunicarse a través de este medio con el familiar migrante? ¿Qué cambios perciben en la comunicación a través de Internet? ¿Cuáles son las motivaciones socio-afectivas que lo inducen a privilegiar una u otra forma de comunicación?

Para tratar de dar respuesta a esos interrogantes se planteó el objetivo general de la investigación: Describir las modificaciones en la comunicación de la red personal del adulto mayor con parientes migrantes, debido al uso de Internet, en los municipios de Filandia, Calarcá, Montenegro, Quimbaya, Armenia y Salento, del departamento del Quindío.

La estrategia metodológica puso énfasis en la caracterización de la red primaria virtual, lo que permitió dar respuesta a las preguntas de investigación y sobre todo determinar, como se da cuenta en este artículo, las posibilidades de Internet en la reconfiguración de la red social primaria del adulto mayor, fracturada por efecto de la migración.

En la investigación se pudo comprobar que hoy la red social primaria del adulto mayor, por efecto de la migración, se ha subdivido en red virtual primaria y red primaria natural; ambas se complementan y gracias a esa reconfiguración la red social primaria cumple con la función de ser el soporte emocional, social y espiritual del adulto mayor.

\subsection{El fenómeno migratorio}

De acuerdo con Ritzer (2001) el concepto de migración está relacionado con el de movilidad y abarca todo movimiento o migración de gentes en el tiempo, en el espacio físico o en la estructura social. Este es un proceso que ha acompañado la historia de la humanidad, pero que en la sociedad contemporánea se ha hecho más frecuente, por condiciones macro-estructurales de tipo político, económico, étnico, religioso, etc. Según las causas o circunstancias que motiven la migración, esta puede ser voluntaria o forzada, interna o externa, individual o colectiva.

La Organización Internacional para las Migraciones (OIM, 2011) informa que a pesar de la persistencia de los efectos de la crisis económica mundial, el número total de migrantes en todo el mundo no ha disminuido en los últimos años; solo hubo una cierta reducción de las corrientes migratorias hacia los países desarrollados durante la crisis, pero no se registraron cambios trascendentales en los modelos de movimiento de personas. En España, por ejemplo, se pasó de 692.228 migrantes en 2008, a 469.342 en 2009.

En el 2010, se estimaba que el número total de migrantes internacionales en el mundo era de 214 millones de personas. Los Estados Unidos de América siguen siendo el principal país de destino, con una población hispana que prácticamente se ha duplicado en los últimos diez años. Las remesas que se envían desde los Estados Unidos de América permanecieron relativamente estables durante la crisis económica y se prevé que sigan aumentado tras la recuperación económica. 
Con respecto a América Latina, Cárdenas \& Mejía (2006) afirman que a partir de 1999 se presentó una verdadera diáspora de latinoamericanos hacía España. El flujo desde Ecuador fue el más importante, con un promedio anual de 51 mil personas, durante el periodo 1998-2004. El flujo de argentinos después de la crisis económica y política en el 2001, se incrementó considerablemente, así como de bolivianos que ocuparon el primer lugar en 2004.

La movilidad intrarregional en América Latina ha adquirido, de igual manera, una importancia mayor, con una tendencia creciente en el registro de emigrantes que no provienen de la región, sino principalmente de países africanos o asiáticos, lo que ha aumentado los conflictos ya existentes con la migración regional, como ocurrió en Argentina en el 2010, cuando un grupo de migrantes principalmente de Bolivia, el Paraguay y Perú fue atacado por grupos de argentinos a raíz de la ocupación de un parque en Buenos Aires.

Es indudable que las migraciones desde la región, tanto internas como hacia el exterior, han contribuido al alivio de problemas estructurales como el desempleo, y las carencias en necesidades básicas, debidas, entre otros factores, al rigor de las políticas económicas que imponen los organismos internacionales y la corrupción interna, que obligan a los países latinoamericanos, como lo explica Bello:

A reducir los alcances de las políticas sociales, a privatizar instituciones públicas, a disminuir su capacidad operativa, a flexibilizar las relaciones laborales; se pierden los mecanismos de regulación de la seguridad social, se disminuye la capacidad adquisitiva de los salarios de los trabajadores, se privatizan los servicios sociales de salud y educación, se elevan las tarifas de agua, electricidad y telefonía, se establecen impuestos restrictivos. (...) Estos aspectos han conducido a que a los altos índices de pobreza de los sectores históricamente excluidos se sumen índices de empobrecimiento paulatino de las clases medias. Sin duda, esta situación impulsa la movilidad de población, dado que las restricciones para el ejercicio de los derechos económicos y sociales terminan minando el derecho a permanecer, el derecho a no migrar. (Bello, 2005, p. 72-73).

En el caso de Colombia, los primeros flujos migratorios se dieron hacia la década de los 60 del siglo pasado, primordialmente hacia los Estados Unidos. En los años 80 se dirigieron hacia Venezuela por el auge de la economía petrolera, los cambios en la legislación de inmigración de Estados Unidos y posteriormente el narcotráfico, pero a partir de la segunda mitad de la década de los noventa se experimentó una aceleración sin precedentes en los flujos migratorios atribuible a dos factores: la crisis económica de fin de siglo y la intensificación del conflicto armado; el destino preferido fue España.

Sin embargo, hay que aclarar que la migración colombiana está determinada no solo por la migración internacional, sino por la interna, debido al conflicto armado, lo que ha llevado al país a ocupar un doloroso primer puesto en el ámbito mundial en número de personas en situación de desplazamiento forzado, además, es grave el problema de la trata de personas que como migración irregular reviste el proceso migratorio colombiano de una gran complejidad. 
De acuerdo con los datos presentados por la OIM (2013), que tienen como base el anuario estadístico del 2008, había para ese año 4.167 .388 colombianos en el exterior, aproximadamente el 9 \% del total de la población. Estados Unidos seguía siendo el país con mayor presencia de colombianos, con 722.269 personas, Venezuela tenía presencia de 295.829, Ecuador de 195.764 y España de 177.413. Llama la atención la feminización del proceso migratorio colombiano: $51.4 \%$ de los migrantes fueron mujeres.

Hasta el 2008 se observa aún un gran predominio de la emigración frente al retorno, pero esa diferencia ha comenzado a decrecer por el interés de mantener los lazos familiares, el endurecimiento de las políticas de migración y la situación económica, en particular en Estados Unidos y España, los principales destinos de los colombianos; esto ha hecho que la posibilidad de retornados sea mayor que la de emigrantes.

Aunque no hay dudas, según el Observatorio Colombiano de Migraciones (2009), sobre la naturaleza económica de las migraciones colombianas, es paradójico encontrar el poco peso que ese mismo motivo, y ante la crisis económica, tiene sobre el retorno, pues lo que se argumenta son motivos familiares. También llama la atención el poco peso que tiene dentro de las migraciones del país el factor de seguridad: la proporción de colombianos que migran por este motivo es de, más o menos, el $10 \%$.

El Eje Cafetero, también conocido como Triángulo del Café, al que pertenece el departamento del Quindío, lugar de la investigación que se presenta, tiene uno de los mayores índices de migración del país, relacionado con sus altos niveles de desempleo, cercanos al 18 \%. Los estudios de Garay E Rodríguez (2005) muestran que los migrantes de esta región se encuentran en un rango de edad de entre 25 a 35 años, de estratos medios y bajos, con nivel educativo de secundaria completa o universitario, que tienden a viajar solos(as) y por razones laborales. De cada tres mujeres que emigraron del país después del 2000, una nació en el Eje Cafetero, madres, tías, amigas, vecinas o conocidas que en su mayoría han emigrado a España, donde sin duda el idioma es el factor determinante.

Para el 2006 el Eje Cafetero, era la región del país con la más alta proporción de hogares con alguno de sus miembros en el exterior (Mejía, 2006), tendencia que ha continuado porque, claramente se identificó en los hogares de los 100 adultos mayores entrevistados, en el caso estudiado, que el $44 \%$ de sus familiares ha emigrado hace menos de 6 años.

Las últimas tendencias de migración de la región revelan que se retorna de España; el 70 \% de los retornados proviene de ese país, pero se emigra a Chile, como lo registran los periódicos locales, tras la promesa de encontrar fácilmente trabajo bien remunerado, hasta por 1500 dólares mensuales (La Tarde.com, 2013, octubre 20).

Para el contexto nacional y de acuerdo con investigaciones realizadas en varias universidades y lo discutido en conferencias internacionales, esta situación trae sustanciales modificaciones en el funcionamiento y estructura de las familias; el hecho, por ejemplo, de que haya mujeres enviando dinero a mujeres o mujeres a hombres, ha transformado las relaciones de poder en el interior de la misma. En general, la dinámica familiar se ve comprometida por los ajustes derivados de la migración como cambio de la vivienda, 
convivencia, afectación de la cotidianidad, reacomodación de nuevos roles y rutinas. Los principales cambios se evidencian en las áreas de la comunicación, la afectividad, y el ejercicio de la autoridad; estos hacen notar la ruptura de esquemas pre-establecidos y nuevas formas de relación.

Para los adultos mayores la migración de sus hijos ha significado, en algunos casos, hacerse cargo de los nietos, enfrentando los problemas propios de la crianza, agravados por la distancia generacional. En la presente investigación se halló que un 23 \% de los adultos mayores ha debido encargarse del cuidado de sus nietos; sin embargo, manifestaron no haber experimentado cambios fundamentales en cuanto al reconocimiento de su autoridad como padres o abuelos. Antes y después del hecho migratorio se han sentido respetados; en ese aspecto la migración del familiar no produjo ningún cambio fundamental.

Los mayores costos que asume el adulto mayor son los emocionales. Independientemente de su situación económica todos manifestaron cambios afectivos como soledad, tristeza, falta de cuidado y apoyo en la vejez, a pesar del respaldo económico que representa el familiar migrante, que en su mayoría corresponde a un hijo.

De allí que sea válida la preocupación por la calidad de vida del adulto mayor, con relación a la comunicación que establece con su red primaria, que en su caso, es un apoyo trascendental por su situación de vulnerabilidad, acrecentada por el fenómeno migratorio. Hay que tener en cuenta que en las familias migrantes la ruptura de la red social primaria crea problemas que deben ser subsanados, como lo plantean Soto et al. (2006): "La separación de la familia, hijos, esposo, padres; de la tierra, la luz, los olores, la música, la comida, desencadena una serie de duelos difíciles de elaborar" (p. 3).

\subsection{Internet y redes sociales. Su incidencia en los procesos migratorios}

Reconocer cómo se reacomodan las redes sociales primarias fracturadas por la migración, gracias a la mediación de Internet, es una necesidad en una época como la nuestra, en la que las relaciones humanas han entrado en crisis debido a la individuación y desagregación de la sociedad. El asunto es que la condición migratoria se vive hoy en un contexto comunicacional en el cual, como lo explica Castells (2009), "Internet en sus distintas aplicaciones, es el tejido de la comunicación de nuestras vidas: para el trabajo, los contactos personales, la información, el entretenimiento, los servicios públicos, la política y la religión" (p.100).

Las tecnologías de la información y la comunicación [TIC], entre ellas Internet, entendidas como la "imitación de la interacción por parte de un sistema mecánico o electrónico que contemple como objetivo principal o colateral también la función de comunicación con un usuario o varios usuarios" (Narváez, 2002, p. 63), y que se basan siguiendo a Castells (2009), en la "digitalización de la comunicación, la interconexión de ordenadores, el software avanzado, la mayor capacidad de transmisión por banda ancha y la omnipresente comunicación local-global por redes inalámbricas de manera creciente con acceso a internet" (p. 89), han hecho que la comunicación interpersonal electrónica, esté presente en todo lo que hacemos, en cualquier lugar y momento y, por consiguiente, han revolucionado la comunicación humana. 
El Ministerio de Tecnologías de la Información y la Comunicación de Colombia informó que para el segundo trimestre del 2011 había en Colombia 5.522.035 suscriptores de Internet, correspondiendo a un 12 \% de la población del país, en comparación con el 4.5 \% en el año 2002, lo que evidencia el crecimiento constante de acceso a la red.

El cubrimiento para el Departamento del Quindío es de un 6.5 \% de la población que, aunque lejos de ser una cifra de cubrimiento ideal, hay que tener en cuenta la facilidad de acceso a través de sitios comerciales que prestan el servicio en casi todos los municipios y a muy bajo costo. Sin embargo, como lo advierte Castells (2009), en los análisis sobre Internet y las comunicaciones inalámbricas, debe considerarse que:

Existe una diferencia abismal en el acceso a la banda ancha y las brechas educativas en cuanto a la capacidad para manejar una cultura digital [que] tienden a reproducir y a ampliar las estructuras de dominación social por clase, etnia, raza, edad y sexo entre países y dentro de cada país (p. 91).

La exclusión digital es sinónimo de marginación social, y de ahí la necesidad de recursos y capacitación tecnológica para las personas adultas, lo que implica reflexionar sobre el papel del Estado en relación con la falta de mecanismos de información y enseñanza a los adultos, más si se tiene en cuenta que en investigaciones realizadas tanto en Europa como en Latinoamérica se advierte que existe una barrera mental que predispone a los adultos mayores para no utilizar las TIC, aunque no dejan de observarse tendencias sobre un uso cada vez más frecuente de la Internet, el celular y los mensajes cortos de texto. Entre los adultos mayores, las mujeres mayores son las que más usan estas tecnologías.

Sin embargo, como lo advierte Machado (2000),

Lo más importante es observar que determinados instrumentos, procesos o soportes que las nuevas tecnologías hacen posible, tienen repercusión en los sistemas de vida y de pensamiento humanos, en su capacidad de imaginar y en sus formas de percibir el mundo (p. 241).

Esta reflexión conduce a la inquietud sobre si los adultos mayores, en su condición de inmigrantes digitales, según la categorización propuesta por Prensky (2001), y ante la ne cesidad de comunicación con su familiar migrante, han podido apropiarse de las mismas y asumir los cambios culturales que conllevan.

Por otro lado, el auge de Internet y sus posibilidades de interacción han puesto en primer plano el debate sobre las redes sociales. El concepto ha ganado relevancia pero tiende a ser utilizado preferentemente para referirse a redes sociales electrónicas, lo que ha causado confusión con respecto al significado de las mismas en la cultura, ya que es necesario tener presente que siempre han formado parte de la naturaleza gregaria de los seres humanos.

En general, las redes sociales se entienden como "un sistema humano abierto, cooperativo y de propósito constructivo que a través de un intercambio dinámico (de energía, materia e información) de sus integrantes permiten la potenciación de los recursos que estos poseen", Reales, Bohórquez \& Rueda, (1993), (citados por Madariaga, Abello \& Sierra, 
2003, p.14), por lo que se hacen indispensables para el sano desarrollo social y psicológico de cualquier persona, tanto así que la Organización Mundial de la Salud [OMS] las considera un aspecto vital para mantener una vida sana, pues les dan a sus participantes no solo sentido de pertenencia sino redes de apoyo para superar las dificultades.

Las redes sociales, en su función comunicacional, conforman el tejido humano y facilitan la transmisión y evolución del conocimiento ya que, gracias a ellas, cada generación recoge los aprendizajes de las generaciones precedentes y aporta los suyos para dejárselos a las generaciones futuras. Los sociólogos, que son los que más han estudiado este tema están de acuerdo en que son más eficientes en situaciones dramáticas de pobreza en los que prima la desestructuración social; en casos así, las necesidades hacen que las personas se encuentren y comiencen a generar aportes individuales que, en medio de las carencias, permiten reunir los elementos requeridos para sobrevivir. "Esto debido a que el atributo fundamental de la red es precisamente la construcción de interacciones para la resolución de problemas y la satisfacción de necesidades, dentro de la participación social" (Madarriaga et al., 2003, p.17).

Pero no solo en situaciones de pobreza o en catástrofes se activan las redes de apoyo, sino también cuando se generan cambios dentro de los sistemas establecidos, como es el caso de la desestructuración de la red social primaria cuando un miembro debe marcharse a otra ciudad o a otro país. En esa situación la red misma crea mecanismos para continuar con su existencia y es aquí donde Internet ha entrado a jugar un papel de vital importancia para recomponer la red social primaria de las familias afectadas por la migración, pues ofrece una variedad de alternativas comunicacionales que amplían de manera insospechada las oportunidades de comunicación y contacto, asunto en el cual se interesó el trabajo de investigación que se presenta.

En ese sentido y desde la perspectiva de Guarnizo (2006), el fenómeno migratorio

[...] se entiende hoy como un proceso dinámico de construcción y reconstrucción de redes sociales que estructuran la movilidad espacial y la vida laboral, social, cultural y política tanto de la población migrante como de familiares, amigos y comunidades en los países de origen y destino (p. 81).

Gracias a esas posibilidades los migrantes no rompen los lazos afectivos, culturales y económicos con sus comunidades de origen; por el contrario, conservan estrechas relaciones familiares y de amistad que conlleva un interesante proceso de intercambio cultural, donde no solo los migrantes mantienen sus valores y principios identitarios sino que permean a sus regiones de origen, con diversas propuestas culturales, tomadas de los países receptores, intensificando procesos de globalización cultural.

\section{Metodología}

Para la investigación se utilizó el método etnográfico de enfoque cualitativo. De acuerdo con esta particularidad se desarrollaron las 4 dimensiones que le son propias: exploratoria, descriptiva, categorial, e interpretativa y de teorización. 
La dimensión exploratoria, estuvo encaminada a la búsqueda bibliográfica relacionada con el tema a investigar y a la identificación de los adultos mayores que en cada municipio cumplieran con los requisitos de la muestra.

La unidad de análisis estuvo conformada por 100 adultos mayores: 40 para la capital del Quindío, Armenia, por tener un número mayor de esta población y 10 para cada uno de los demás municipios: Salento, Quimbaya, Montenegro, Filandia, Calarcá. Los municipios fueron escogidos por ser los de mayor índice de migración. Se acudió a la muestra intencional: adultos mayores de 60 años, con un familiar migrante.

La dimensión descriptiva tuvo que ver con la aplicación del instrumento de recolección de información, previa aplicación de la prueba piloto para identificar posibles falencias en el diseño de las preguntas, en el desarrollo y en el tiempo de duración de la entrevista, en la claridad de las preguntas y en el manejo del tema a investigar por parte de los entrevistadores, estudiantes de último semestre del programa de Comunicación Social-Periodismo de la Universidad del Quindío. Se utilizó, la entrevista semi-estructurada de preguntas guía, con grabación sonora y se emplearon formatos para el registro de la información y su sistematización.

En coherencia con los objetivos de la investigación, la entrevista se diseñó para dar cuenta de las características de la red virtual primaria conformada por el adulto mayor tras el proceso migratorio. Se indagó por cuatro características: competencias digitales, estructura de la red, funciones y atributos de vínculo.

Las competencias digitales y la estructura de la red permitieron identificar el nivel de autonomía del adulto mayor en el manejo de la herramienta y determinar la apropiación que tiene con respecto a este tipo de comunicación. Las funciones y atributos de vínculo permitieron identificar las motivaciones socio-afectivas que llevan al adulto mayor a establecer la comunicación virtual y, desde sus percepciones, establecer el nivel de satisfacción con la misma.

Para el tratamiento de la información, las entrevistas se organizaron por códigos numéricos, como forma de identificar los datos desde los actores entrevistados, el lugar y las categorías con relación a la pregunta realizada. Por ejemplo, en la entrevista marcada con el 0.2. 3 B-P.4, el 02 corresponde al número asignado al municipio, el 3 al número de la entrevista, la letra B a la categoría y P.4 al número de la pregunta.

Para la dimensión de categorización que consistió en analizar de manera detallada y minuciosa las grabaciones sonoras de las entrevistas y las transcripciones de las mismas, con el fin de identificar las categorías y subcategorías en la búsqueda de un entretejido categorial del cual emergieran los diferentes significados de los hechos narrados, se acudió a la propuesta metodológica de Strauss, A. \& Corbin, J., (2002), la Teoría Fundamentada, con sus tres componentes de Codificación: la Codificación Abierta que permitió descomponer los datos y realizar comparaciones; la Codificación Axial, que contribuyó a relacionar categorías y subcategorías y la Codificación Selectiva, con el fin de de integrar y refinar las categorías. 
La interpretación y teorización de los resultados se realizó desde los hallazgos de las dimensiones anteriores, con los aportes de la teoría y las observaciones de los investigadores en todo el proceso. En esta dimensión se consolidó el corpus de la investigación para dar cuenta de las posibilidades que ofrece Internet en la reconfiguración de la red social primaria y las consecuentes modificaciones que esta circunstancia trae en la comunicación del adulto mayor con su red social primaria.

\section{Resultados}

\subsection{Competencias digitales en los adultos mayores}

Por competencias digitales se entienden las habilidades que se deben desarrollar para el manejo autónomo de los artefactos o herramientas que permiten el acceso a las TIC. Con relación a esta categoría, en el proyecto se indagó por el tipo de herramienta tecnológica usada, el tiempo de uso de la misma y las fases de aprehensión en el manejo de la herramienta.

\subsubsection{Tipo de herramienta tecnológica usada}

Teniendo en consideración el factor multimedia, la herramienta de comunicación virtual más utilizada por los adultos mayores, para comunicarse con sus parientes es la videoconferencia, gracias a las libertades expresivas y experiencias afectivas que ofrece, tales como la conjugación de sonido y vídeo, sincronía y duración, y la sensación de estar en el mismo lugar con su pariente.

Fueron comunes expresiones como: "Es increíble poderla ver por la pantalla" (04.2 D-P.28.), "...sí, mis momentos más felices son cuando la puedo ver a ella por la cámara" (04.10 B-P. 12). Es de anotar que especialmente este tipo de herramienta se valora por la calidad de la comunicación que permite: "La comunicación es muy buena, afianzamos mucho los vínculos familiares, nos contamos todo y sí es muy abierta" (04.9 B-P.10), "[Esta comunicación] es mejor, pues encontramos más temas de que hablar, cosa que no hacíamos acá" (04.2 B-P.10). "Nos contamos anécdotas, nos contamos lo que nos pasa, lo que hacemos, hasta hemos llegado a tomar frente al computador" (04.9 B-P.8).

Otro factor determinante para optar por este tipo comunicación es la falta de destreza para escribir en el teclado, como muchos de ellos lo afirmaron; de allí que sean muy pocos los que usan el del chat o el correo electrónico. Menos del 25 \% usa una forma de comunicación adicional a la videoconferencia.

Se tiene entonces que Internet, y en este caso específico la videoconferencia, posibilitó trascender las barreras del teléfono tradicional, y por su capacidad de acercamiento, por el disfrute de la voz y la posibilidad de ver y sentir la presencia del familiar, ha proporcionado mayor confianza y seguridad en la comunicación. 


\subsubsection{Tiempo de uso de la herramienta}

La herramienta se usa entre 1 y 3 horas semanales, una o dos veces a la semana. Si se tiene en cuenta la gratificación emocional que les produce la comunicación por video conferencia, el tiempo que dedican al uso de la misma, no es un problema; al contrario, manifiestan que por el cambio de horario, el tiempo no es suficiente. Los adultos mayores anotaron que por la separación familiar y debido a las posibilidades de la herramienta utilizada, la comunicación, a diferencia de la que sostenían antes, cuando el pariente no había migrado, es más fluida y afectuosa.

Manifestaron que la videoconferencia es un medio eficaz para cultivar los lazos familiares y que el encuentro virtual les produce sentimientos de alegría y felicidad, expresiones como: "La distancia ha mejorado la comunicación y nos ha unido más, nos da alegría" (02.2 B-P4), "La comunicación es más abierta, la distancia nos une más" (0.2.3 B-P.4), "La comunicación ha mejorado, porque no estamos cerca... así es la vida" (02.4 B-P.4), "La relación ha mejorado claro.., porque ya está uno más pendiente" (02.5 B-P.4), lo confirman.

\subsubsection{Fases de aprehensión}

Teniendo como referente a Tovar \& Villarraga (2009), que señalan como fases de aprehensión de las nuevas tecnologías "el saber acceder y manejar básicamente la Internet, utilizarla para la solución de problemas y finalmente estar en capacidad de manipularla como herramienta para la producción de información o conocimiento e inclusive aprendizaje" (p. 29), se encontró poca motivación de los adultos mayores para adquirirlas, pues cuentan con familiares o sitios de Internet que resuelven sus dificultades.

Como lo afirman Tovar y Villarraga, son los integrantes de la red personal quienes ejercen mayor influencia sobre el adulto mayor para que este se comunique vía Internet con su pariente migrante; son estos quienes brindan las condiciones técnicas y de aprendizaje.

Adicionalmente, se observó que hay mucho temor en el adulto mayor para enfrentar los retos tecnológicos, "Me da miedo tocar esos aparatos, de pronto se daña porque yo no sé nada de eso" (04.2 A-P.3), y no se exploran todas las posibilidades del medio y de la herramienta: "No lo utilizo [el computador] para nada más, porque ni siquiera lo sé prender" (04.2 D-P.25). Solo en casos de adultos mayores que antes de su jubilación se vieron obligados a manejar la herramienta por asuntos laborales, esta se utiliza con fines de socialización, cultura o salud: "Lo tuve que aprender a manejar por mi trabajo en la notaría ya que todo, hoy en día, es por computador" (04.9 A-P.4), lo que no ocurre cuando se trata, por ejemplo, de amas de casa que no tuvieron vida laboral por fuera de sus hogares.

Un aspecto interesante a considerar es que Internet ha generado un cambio de roles al convertir al joven en maestro del adulto, lo que en no pocas ocasiones genera una relación conflictiva niño-joven / adulto mayor, ante el estereotipo que pesa sobre este, que es considerado lento e incapaz de aprender. 
Esos prejuicios sobre los adultos mayores se encuentran con los que existen sobre los jóvenes, pues se supone que ser joven y saber de tecnología son cosas normales, supuesto que se aceptan sin cuestionar o intentar romper. Por eso, cuando se les preguntó a los adultos mayores si deseaban capacitarse para desarrollar habilidades en el uso de la herramienta, no fue raro encontrar respuestas tales como: "Me gustaría, pero loro viejo ya no aprende" (01.2 B-P.5), "No, para qué si mi hijo me ayuda" (04.2 B-P. 11), o ante la pregunta sobre ¿Cúal ha sido la principal dificultad a la hora de comunicarse vía Internet?, se dieron respuestas como: "pues en realidad ninguna, sino que no me ha interesado [aprender] porque yo llego a una sala y ahí me alistan todo, entonces, pues, no me he propuesto aprender" (04.4 A-P.3).

\subsection{Características estructurales de la red virtual primaria}

Sluzky (1996), (citado por Madariaga, Abello E Sierra, 2003) sugiere que las redes "se pueden analizar en función de tres propiedades: 1. Sus características estructurales, 2. Las funciones de los vínculos y 3. Los atributos de cada vínculo" (p. 29). Sin embargo, en la presente investigación, no se tuvo en cuenta para el análisis de las características estructurales, la propuesta de Sluzky, debido a que las redes estudiadas son muy pequeñas, generalmente compuestas por dos o tres personas: el adulto mayor, su hijo y nieto, para las que difícilmente aplican todos los factores de esta propiedad; además, son redes de carácter simétrico que siempre usan la misma herramienta, videoconferencia, y siguen la misma rutina en el momento de la comunicación.

Baste con anotar, por ejemplo, con respecto al tamaño de la red, en el caso de la red virtual del adulto mayor que este factor no tiene el alcance dado por Sluzky ya que, según este autor, a mayor tamaño mayor posibilidad de que la red permanezca en el tiempo, aunque se pierda la relación entre algunos miembros. Lo que se observó en la red primaria virtual del adulto mayor es que es la fuerza del vínculo familiar la que la mantiene en el tiempo y garantiza la permanencia de sus integrantes, sin importar la cantidad de los mismos.

Por lo tanto, de acuerdo con los intereses del trabajo de investigación, el énfasis se puso en las funciones y los atributos de los vínculos, más que en las características técnicas de la red, porque su estudio otorgaba mayores elementos de juicio para identificar las posibilidades de la red virtual en la reconfiguración de la red primaria y, por consiguiente, su incidencia en la calidad de vida del adulto mayor con respecto a la satisfacción de sus necesidades comunicacionales y afectivas.

Un aspecto que sí se tuvo en cuenta, en cuanto a la estructura de la red, fue la topología, que se refiere a la cadena de comunicación que une los nodos entre sí, con el fin de complementar el conocimiento sobre el nivel de autonomía del adulto mayor en el manejo de la red y la herramienta. Las topologías más utilizadas son: 


\section{Gráfica 1}

\section{Topologías}

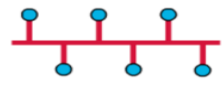

Topología de bus

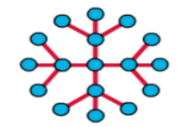

Topología en estrella extendida

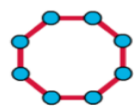

Topología

de anillo

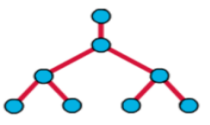

Topología

jerárquica

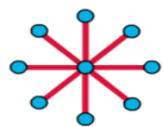

Topología

en estrella

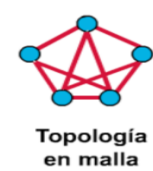

Fuente: www.docstoc.com (2013)

Con respecto a estas topologías, se encontró que los adultos mayores del Quindío, en su gran mayoría, han conformado redes sociales estructuradas desde la topología en estrella, en la cual ellos, como sujetos centrales, estimulan y dan origen al deseo de comunicación, pero hay que aclarar que la iniciativa de crear las cuentas como usuarios de Internet no fue de ellos sino de los parientes migrantes.

Se encontraron algunos pocos casos que respondían a la topología de anillo y sobre todo cuando los parientes migrantes no eran los hijos. Estos se ubicaron en la ciudad de Armenia, capital del departamento y cuando el adulto mayor respondía a alguna de las siguientes condiciones: fue autodidacto en el manejo de la herramienta; se conservaba activo laboralmente o tenía un interés manifiesto en establecer vínculos sociales, hacer amistades por medios virtuales y pertenecer a clubes o grupos. En esas situaciones era el adulto mayor el que impulsaba y dinamizaba la relación entre los nodos, pues las redes sociales virtuales le significaban poder ampliar sus lazos sociales más allá de lo estrictamente familiar, y se sentían motivados por indagar y aprender nuevas alternativas en el uso de la Internet y la red.

\subsection{Funciones de los vínculos en la red virtual primaria}

A partir de lo planteado por Sluzky (1996), (citado por Madariaga, Abello \& Sierra, 2003), se seleccionaron las características de cada propiedad que tuvieran directa relación con el objetivo de identificar las motivaciones socio-afectivas que llevan al adulto mayor a establecer la comunicación virtual.

Las funciones de vínculo que se refieren al tipo de intercambio interpersonal que permiten las redes, según Sluzky, son: compañía social, es decir, la posibilidad que permiten de realizar actividades en conjunto; apoyo emocional, como la posibilidad de realizar acciones que permitan la conexión emocional y una actitud emocional positiva; guía cognitiva y consejos, que consiste en la opción que brinda la red para ofrecer modelos de 
rol social, aclarar expectativas y brindar información; regulación social, que son aquellas acciones que recuerdan y reafirman los roles, así como la distribución de información que permita que las expectativas colectivas se mantengan y se pueda disipar la frustración o violencia colectiva; ayuda material y de servicios como la forma en que la red provee ayuda profesional a través de la interacción de sus miembros y acceso a nuevos contactos, que se refiere a la posibilidad que las redes brindan de abrirse hacia nuevos contactos y ampliar la red personal de sus integrantes.

Aquí se encontró que en el caso de los adultos mayores con parientes migrantes en el Quindío, la función prevalente es la de apoyo emocional, pero la red virtual primaria también cumple con funciones de guía cognitiva y de consejos y de regulación social.

La función de apoyo emocional es la prevalente porque gracias a ella se sostienen los vínculos familiares tan importantes y vitales para el adulto mayor. Con relación a la misma manifestaron sentimientos como: "Me siento muy feliz lal comunicarsel y me da nostalgia al verlos, y saber que están tan lejos y quererlos abrazar" (04.1 B-P 9), "Yo no me acostumbro a su ausencia, es un momento de tristeza y de alegría; cuando hablamos [por videoconferencial lloramos" (04.2 B-P 9).

La característica de vínculo familiar que tiene la red facilita superar, de igual manera, las dificultades que se puedan presentar por la heterogeneidad demográfica: adulto mayor / hijo, adulto mayor / nieto; el encuentro se logra gracias a que los temas frecuentes y comunes de conversación son la salud, la economía del hogar o asuntos personales; esto le ofrece al adulto mayor otro tipo de satisfacción: continuar con su labor de guía espiritual y de consejo, se siente útil, necesitado y amado. Cumple, así, la red virtual primaria con otra función esencial, la de regulación social, ya que el adulto mayor puede reafirmar su rol de padre o abuelo y logra disipar, en algo, la frustración o carencias afectivas ocasionadas por la migración.

\subsection{Los atributos de vínculo de la red virtual primaria}

Con respecto a los atributos de cada vínculo, los clasificar Sluzky en funciones prevalentes que son funciones o combinaciones de funciones que caracterizan de manera dominante el vínculo; multidimensionalidad o versatilidad, referida a cuántas de esas funciones cumple; reciprocidad, si una persona cumple con respecto a otra el mismo tipo de funciones; intensidad o compromiso de la relación, atracción entre los miembros y grado de intimidad; frecuencia de los contactos, a mayor distancia mayor el requerimiento de mantener activo el contacto para mantener la intensidad e historia de la relación, referida al tiempo que hace que se conocen los integrantes de la red y cuál es la experiencia previa de activación del vínculo.

Como ya se planteó, la función prevalente es la de apoyo emocional y, aunque la red virtual primaria cumpla con otras funciones, como las arriba mencionadas, su multidimensionalidad o versatilidad es muy limitada porque por lo general solo se establece contacto con el familiar migrante, lo que reduce al máximo las posibilidades de que otros contactos o nodos potencien el alcance de las funciones ofrecidas por la red. La red virtual primaria es 
manejada como una red privada de carácter horizontal y cerrado. Ninguno de los entrevistados afirmó haber creado nuevas amistades luego de que iniciara el uso de este medio.

Con relación a la reciprocidad, la intensidad o compromiso de la relación y la frecuencia de los contactos son la necesidad de comunicación mutua, migrante /adulto mayor, el grado de consanguinidad y la experiencia previa en la relación familiar, las que los definen. Si la comunicación hijo/padre era buena antes de la migración, la red virtual primaria se activa y se mantiene y en muchos casos, como lo manifestaban los adultos mayores, mejora la comunicación.

Sin embargo, en cuanto a la reciprocidad, se encontró que el adulto mayor responde de manera pasiva a los llamados del pariente que emigró, disfruta del momento de la comunicación y la necesita, pero toda la iniciativa del vínculo está en el pariente migrante, quien define el tiempo para la comunicación y la frecuencia.

\section{Conclusiones}

El acceso a Internet de los adultos mayores con parientes migrantes posibilitó reconfigurar la red primaria (familia, amigos, compañeros), fracturada por efecto de la migración. La red primaria se mantiene hoy, no solo de manera natural sino a través de Internet, subdividiéndose en dos redes complementarias: la red primaria natural y la red primaria virtual.

El adulto mayor se vio impelido a acceder a la red virtual primaria, con el fin de satisfacer sus necesidades afectivas y de comunicación. En esta se participa con el fin mantener, pero no de extender, su red social primaria.

Se hizo evidente la importancia, necesidad y complementariedad de los dos tipos de redes. Ambas, la red virtual primaria y la red primaria natural, cumplen de manera prevalente con la función de Apoyo emocional, y se constituyen en el soporte afectivo, social y espiritual del adulto mayor con parientes migrantes, lo que le da sentido y significado a su nueva condición de vida.

Internet le hizo posible al adulto mayor establecer una comunicación de calidad, afectuosa, duradera, intensa y frecuente. Prefieren las comunicaciones sincrónicas (videoconferencia), porque les da la sensación de estar en contacto directo con el pariente y no les exige la digitación de texto o el manejo de órdenes complicadas sobre la herramienta.

Las posibilidades que no aprovecha el adulto mayor en el caso de la red virtual primaria, como ayuda material y de servicios y acceso a nuevos contactos, son subsanadas por la red social natural, basada en las relaciones personales de cada individuo y en este caso integrada por amigos y familiares no migrantes. La red social natural cumple, de igual manera, funciones de vínculo, esenciales como la compañía social. En su mayoría, los adultos mayores pertenecen a asociaciones filantrópicas o de apoyo a la tercera edad, y/o cuentan con un grupo de amigos con los que se recrean y se proveen de ayuda material o de servicios.

Se encontró que los adultos mayores, por lo general, no dominan las nuevas tecnologías, practicamente no han desarrollado competencias digitales, salvo aquellos que 
llevaron o llevan una vida laboral activa puesto que la exigencia de sus propias ocupaciones los incitaron a acercarse a conocer Internet y otros sistemas de comunicación modernos.

Un aspecto que imposibilita el aprendizaje del uso de Internet y sus herramientas es la dependencia de un tercero, y la prevención hacia su manejo. Esta situación ha obstaculizado que los adultos mayores se apropien de su condición de inmigrantes digitales y así pueden acceder al mundo que ofrecen las nuevas tecnologías en materia comunicativa, afectiva, laboral, de socialización, cultural y de conocimiento, de tal manera que los instrumentos, procesos o soportes que estas hacen posible tengan impacto y repercusión en sus "sistemas de vida y pensamiento (...), en su capacidad de imaginar y en sus formas de percibir el mundo", retomando a Machado (2000, p.241).

Son los parientes que viven con el adulto mayor, los encargados de manejar el computador y, por lo tanto, los que dominan de alguna manera la comunicación. Los horarios de encuentro se definen no solo por el tiempo libre que tenga el pariente migrante sino por la disponibilidad del pariente cercano, lo que ha implicado, ademas, que la necesidad del adulto mayor de establecer la comunicación virtual cambie su rol, pues pasa de tener una posición relevante dentro de la educación de sus nietos o hijos a ser el educado por estos.

El adulto mayor no cumple tareas de nodo activo en la red virtual primaria debido a las limitaciones anotadas. A pesar de ello, se sienten motivados a usar la red y se han vuelto dependientes de la misma por la relación de feedback y reciprocidad que ofrece este tipo de comunicación.

\section{Referencias bibliográficas}

Bello, M. N. (2005). Migraciones, redes sociales y ciudadanía. Aportes para la definición de políticas migratorias en Colombia, Ecuador y España. Otras Visiones desde la Cultura. Bogotá: Convenio Andrés Bello, Universidad Javeriana

Cárdenas, M., E Mejía, C. (2006). Migraciones internacionales en Colombia ¿qué sabemos? Santiago de Chile: CEPAL. Recuperado el 14 de abril, 2011. Disponible en http://www.iadb.org/intal/intalcdi/ pe/2009/02989.pdf

Castells, M. (2009). Comunicación y poder. Madrid: Alianza Editorial.

Flórez, P., (2013, 21 de octubre). ¿Chile, una nueva promesa para el migrante pereirano? La Tarde.com. Recuperado el 20 de octubre de 2013. Disponible en http://www.latarde.com/noticias/risaralda/103953chile-una-nueva-promesa-para-el-migrante-pereirano?quicktabs_ $3=0$

Garay, L. J E Rodríguez, A. (2005). Características socioeconómicas de la población emigrante internacional ubicada en el AMCO. En Memorias del Seminario Migración Internacional, el impacto y las tendencias de las remesas en Colombia, (pp. 33-40). Bogotá: OIM. Recuperado el 11 de Mayo, 2011. Disponible en http://www.remesasydesarrollo.org/uploads/media/Migracion _ Internacional_el_Impacto_y_las_Tendencias_de_las_Remesas_en_Colombia.pdf

Guarnizo, L. E. (2006). Migración, globalización y sociedad: teorías y tendencias en el siglo XX. En G. Ardila (Ed.), Colombia, migraciones transnacionalismo y desplazamiento (pp.261-276). Bogotá: Unibiblos. Recuperado el 15 de octubre de 2011. Disponible en http://www.bdigital.unal.edu.co/785/3/257 _ _ ${ }_{-}^{2}{ }_{-}$Capi_l.pdf 
Machado, A. (2000). El paisaje mediático. Sobre el desafió de las poéticas tecnológicas. Buenos Aires: Libros Rojos.

Madarriaga Orozco, C., Abello Llanos R., E Sierra García, O. (2003). Redes sociales. Infancia, familia y comunidad. Barranquilla: Universidad del Norte.

Mejía Ochoa, W. (2006). Las migraciones como posible tema para un informe de desarrollo humano en el Eje Cafetero. Programa de las Naciones Unidas para el Desarrollo [PNUD]. Perspectivas para un segundo informe de Desarrollo Humano en el Eje Cafetero. Recuperado el 23 de marzo del 2012. Disponible en http://www.quindio.gov.co/home/docs/general/ejecafeteromigracionesydesarrollo.pdf

Narváez, A. (2002). Puentes tecnológicos, abismos sociales. Manizales: Centro de publicaciones Universidad de Manizales

Observatorio Colombiano de Migraciones. (2009). Presentación Encuesta Nacional de Migración Internacional y Remesas. Recuperado el 8 de agosto de 2011. Disponible en http://www.humanas.org.co/ archivos/InformeEncuesta.pdf

Organización Internacional para las Migraciones. (2011). Informe para las migraciones en el mundo 2011. Recuperado el 15 de febrero de 2012. Disponible en http://publications.iom.int/bookstore/ free/WMR2011_Spanish.pdf

Organización Internacional para las Migraciones. (2013). Datos y cifras sobre migración colombiana. Recuperado el 20 de octubre de 2013. Disponible en http://www.oim.org.co/migracion-colombiana/ datos-sobre-migracion-colombiana.html

Prensky, M. (2001). On the Horizon. En: MCB University Press, Vol. 9 N. ${ }^{\circ}$ 6. Recuperado el 20 de abril, 2012. Disponible en http://ceipbeataines.org/pluginfile.php/582/mod_resource/content/2/nativos_inmigrantes_digitales_marc_prensky_beata_ines.pdf

Ritzer, G. (2001). Teoría Sociológica Clásica (3. a ed). Madrid: McGraw-Hill

Soto Peña, O., Montero Navarro, J., E Sánchez Bocanegra, C. (Noviembre, 2006). Comunicación y redes sociales. Herramientas de software libre para afrontar el reto migratorio. Ponencia presentada en el 2. ${ }^{\circ}$ Encuentro en Línea de Educación y Software Libre. EDUSOL. Recuperado el 5 de abril de 2012. Disponible en http://edusol.info/es/system/files/Comunicacion _ y _ redes _ sociales.pdf

Strauss, A. E Corbin, J. (2002). Bases de la investigación cualitativa. Medellín: Universidad de Antioquía.

Tovar Pinilla, A. E Villarraga Olaya, C. (2009). Inclusión de las NTC en la mediación social de los adultos mayores como usuarios virtuales. (Tesis de pregrado). Universidad Sergio Arboleda. Bogotá. Recuperado el 11 de octubre de 2012. Disponible en http://www.usergioarboleda.edu.co/investigacion-comunicacion/documentos_ _ edicion _ 1/adultos-internet.pdf 